Avances y desafíos de un modelo de universidad pública en tiempos de pandemia.

Fernando Tauber

Revista ES (en y sobre Educación Superior)

Vol.1, N¹-2 / Fecha de publicación: 27/12/2021

e-ISSN: 2718-6539

https://revistas.unlp.edu.ar/ES/index

IIES - Facultad de Odontología

DOI: https://doi.org/10.24215/27186539e039

\title{
Avances y desafíos de un modelo de universidad pública en tiempos de pandemia.
}

Advances and challenges of a public university model in times of pandemic.

Avanços e desafios de um modelo de universidade pública em tempos de pandemia.

Dr. Arq. Fernando Tauber

Presidente de la UNLP 2018-2022.

fernando.tauber@presi.unlp.edu.ar 


\section{Resumen}

Estado de la gestión de la UNLP a través de una síntesis operativa del Plan Estratégico, el Plan de Desarrollo Institucional y la información de las áreas de gobierno.

\section{Abstract}

Status of the management of the UNLP through an operational synthesis of the Strategic Plan, the Institutional Development Plan and the information of the government areas.

\section{Resumo}

Situação da gestão da UNLP por meio de síntese operacional do Plano Estratégico, do Plano de Desenvolvimento Institucional e das informações das áreas governamentais.

\section{Palabras clave}

Gestión, Plan Estratégico, Planeamiento, Desarrollo, Universidad

\section{Keywords}

Management, Strategic Plan, Planning, Development, University

\section{Palavras chave}

Gestão, Plano Estratégico, Planejamento, Desenvolvimento, Universidade

Cada vez que das un paso adelante, estás destinado a perturbar algo. Agitas el aire mientras avanzas, levantas polvo, alteras el suelo. Vas atropellando cosas. 
Cuando una sociedad entera avanza, ese atropello se hace en una escala mucho mayor; y cada cosa que trastornes, los intereses creados que quieras suprimir, todo se convierte en un obstáculo (a vencer, siempre a vencer).

MAHATMA GANDHI

\section{Los objetivos de nuestra Universidad Pública}

Las universidades de América Latina y el Caribe acordamos hace tiempo que para nosotros la educación superior es un bien público y social, un derecho humano universal y una responsabilidad del Estado. Desde la década del 90 defendimos esa visión en cada debate dado en los foros educativos mundiales contraponiendo este modelo al representado en la mayoría de los países del resto del mundo que entiende el acceso a los conocimientos superiores como un bien selectivo, transable en el mercado.

La expectativa de nuestro desarrollo como país y nuestro progreso individual estuvieron "siempre" sustentados en la igualdad universal de oportunidades basada en principios fundamentales de inclusión. El ingreso irrestricto y la gratuidad de la enseñanza universitaria garantizadas definitivamente en la Argentina por la ley 27.204 sancionada el 28 de octubre y promulgada por la Presidenta Cristina Fernández de Kirchner el 9 de noviembre de 2015, ya nacieron en las ideas de la Generación del '80 -Sarmiento, Avellaneda, Rocaplasmadas en 1884 en la Ley 1.420 de Educación Común con los preceptos de "laica, obligatoria y gratuita" para la educación primaria, extendida luego a la secundaria. Se plasmaron en el ideario de la Reforma Universitaria de 1918 y se concretaron inicialmente en el Decreto 29.337 que firmara el Presidente Juan D. Perón el 22 de noviembre de 1949. 
Hoy a nadie se le ocurre proponer revisar los conceptos de gratuidad para la educación inicial, primaria y secundaria y debemos asegurarnos que en esa línea no queden bolsones de resistencia a la gratuidad de la enseñanza universitaria. El modelo nacional, largamente centenario, impone el derecho a la educación de todo el que esté dispuesto a hacer el esfuerzo de superarse, adquiriendo y acreditando más conocimientos.

La universidad debe ser un espacio para tener ideas y discutirlas, alejada del pensamiento único, promotora del pensamiento diverso, original y propio; caracterizada por estar llena de inquietudes, rechazos, audacias, escrúpulos y esperanzas que custodian el comportamiento ético, transparente, republicano y democrático de sus miembros.

Debe ser firmemente inclusiva y entender a la educación como una línea ininterrumpida e ininterrumpible que no corresponde que se corte por una falla del sistema que frene el proceso de toma de conocimientos de aquel que quiere seguir sumándolos por un fracaso, dificultad o deficiencia circunstancial. Si un estudiante no sabe lo suficiente para avanzar a un estadio superior en el proceso de su formación, pero tiene la firme voluntad de resolver sus déficits esforzándose en sus estudios, la universidad debe darle siempre esa oportunidad. No puede dejarlo flotando en un limbo que no le permita ni retroceder ni avanzar. $\mathrm{Y}$ esto debe suceder desde el ingreso hasta la finalización de sus estudios.

Esto no significa resignar calidad (ni pertinencia, ni la siempre bien defendida búsqueda de la excelencia), por el contrario, significa agregarle responsabilidades al Sistema. Ese es el verdadero "contrato" que firmamos cuando este modelo fue concebido para desarrollar al país y multiplicar las oportunidades para el progreso colectivo. La calidad y la masividad, asociada al 
esfuerzo y al compromiso son un conjunto indisoluble. Si existe uno de estos factores, necesariamente deben existir los otros en la universidad pública argentina.

No puede considerarse realmente de calidad la educación superior de un país que acepta dejar afuera a la mayoría de la población y no apunta a ofrecerle oportunidades de acceso. La calidad incluye a la ética y a la conciencia social y es claro que cuando el acceso a la educación superior se limita a pocos, ella tiene características que la asemejan a un bien de mercado y no a un derecho universal.

Los principios de la Reforma de 1918, nacidos en la Universidad Nacional de Córdoba, quizás la última gran revolución en la educación superior de alcance continental, fueron asimilados inmediatamente por las Universidades Nacionales de La Plata y Buenos Aires y las Provinciales de Tucumán y El Litoral (únicas en ese momento en la Argentina) y se propagaron inmediatamente por América Latina y el Caribe.

Sus postulados de autonomía, cogobierno, libertad de cátedra, gratuidad, ingreso irrestricto, ingreso a la docencia por concurso, extensión universitaria y concebir a la Universidad como el ámbito natural de debate de los temas que le importan e interesan a la Sociedad, señalaron el camino ya centenario para la construcción de un modelo de inclusión, todavía incompleto en su concreción en vastos territorios de nuestro continente, que se sostiene en la enseñanza, la investigación y la extensión universitaria.

Sin embargo, las tremendas asimetrías sociales en contar con oportunidades de progreso y la permanente y creciente defensa de la construcción de nuestros países a partir de decisiones democráticas y soberanas, imponen 
sumar a esos postulados básicos a la producción (sostenida en el desarrollo de proyectos científico tecnológicos y su transferencia) y al trabajo (impulsado por la innovación, el emprendedorismo y el fortalecimiento de nuestros recursos humanos formados en todos los niveles, incluso el de educación formal alternativa, desde la propia gestión universitaria).

Necesitamos que la Universidad Pública reafirme su condición de herramienta para la transformación positiva de nuestra Patria y de progreso generalizado de sus habitantes, fundamento de las decisiones soberanas y fábrica de oportunidades para el conjunto de nuestra sociedad. Más aún en esta situación de pandemia que nos desafía e interpela ¿puede esta o cualquier coyuntura, aunque amenace a ser recurrente, hacernos arriar las banderas que aprendimos a levantar con enorme sacrificio, pero también con firme convicción y compromiso?

\section{El modelo de universidad en la Universidad Nacional de La Plata}

Este modelo transparente e inclusivo comprende brindar los conocimientos específicos para concebir al mejor profesional, pero también generar el ambiente imprescindible para contribuir fuertemente a dotarlo de los valores fundamentales para consolidarlo como el mejor ciudadano, pleno de solidaridad, consciente de su responsabilidad para los que menos tienen, tolerante hasta la exageración (para algunos), sabiendo que el debate, el pensamiento diferente y las culturas generacionales siempre son más que el silencio homogéneo e indiferente.

Convencidos que la democracia es un bien indispensable para poder alcanzar nuestros objetivos más preciados, que nos costó mucho alcanzarla y sostenerla y que no es una condición natural inextinguible si no la protegemos y la 
mejoramos con más calidad y más derechos; que la defensa firme del medio ambiente es una suerte de conciencia para dejarle a nuestros hijos y a sus hijos un mundo mejor que el que recibimos y en el que vivimos; que los derechos humanos no sólo significan la custodia de nuestra memoria en la búsqueda de la verdad y de la justicia para saldar las deudas de un pasado injusto, brutal y doloroso y un presente inquietante, sino que acompañan cada acto de nuestra vida, basados en la convivencia, en la mentada búsqueda de la igualdad, en el reconocimiento del diferente, de las minorías y de cada uno de nosotros como un par.

En un mundo donde el conocimiento, la ciencia y la tecnología juegan un papel de primer orden, el desarrollo y el fortalecimiento de la Universidad Pública, tal como la concebimos, antes, durante y después de una pandemia, constituyen un elemento insustituible para el avance social, la generación colectiva de riqueza, el fortalecimiento de las identidades culturales, la cohesión social, la lucha contra la pobreza y el hambre, la prevención del cambio climático y la crisis energética, así como para la promoción de una cultura de paz, en la convicción que la búsqueda de la igualdad y la inclusión, a partir de una sociedad con igualdad de oportunidades de acceso al conocimiento, es indispensable para consolidar una Patria soberana.

Para entender la base conceptual que fundamenta la razón de ser y reacción de la UNLP ante esta circunstancia inesperada y repentina de la pandemia 2020-21, y la construcción sólida de un futuro "normalizado", nuestra universidad tiene y confirma como objetivos primordiales:

1- Formar estudiantes en el pregrado, grado y posgrado volviéndolos graduados firmes en sus conocimientos específicos y en sus condiciones y convicciones ciudadanas, comprometidos siempre con su comunidad, 
buscando el crecimiento constante de la matrícula, la permanencia con avances sostenidos y continuos en sus carreras que permitan su culminación en tiempos acordes y conscientes del esfuerzo social y personal necesario de hacer para alcanzar su graduación. No debemos conformarnos con sospechar que el sólo paso por la universidad transforma positivamente al individuo. El país necesita de profesionales probos que apuntalen su transformación y la universidad pública tiene la alta responsabilidad de proporcionarlos.

Este supuesto "del sólo paso" puede valer para el conjunto del colectivo social. No para quienes tenemos la responsabilidad de educar y ayudar a formar. Para nosotros, cada estudiante que abandona es un fracaso que debemos impedir con nuestro esfuerzo.

2- La UNLP también se propone producir conocimientos útiles para el desarrollo del país y el bienestar de la humanidad, con una agenda científica y tecnológica convergente con las necesidades de nuestra sociedad y las demandas de nuestro país para su desarrollo soberano. Entendemos que nunca habrá soberanía sin conocimiento propio y popular. El hambre, la pobreza, las enfermedades y plagas que nos aquejan y toda demanda insatisfecha para una mejor calidad de vida, la calidad y preservación de los recursos naturales, la producción sustentable y responsable y la colaboración con toda política pública apuntada a la necesidad social y el desarrollo nacional y regional, constituyen las grandes líneas para una investigación orientada, emergente de una sólida investigación básica y promotora de una investigación aplicada transferible y funcional a la concreción de estas metas.

Para esto debe proteger y crecer en sus recursos humanos formados que son su mayor capital. La infraestructura y el equipamiento adecuado y los insumos indispensables son fundamentales, pero también cáscaras vacías de contenido 
si no contamos con un capital humano capacitado y dotado de los valores en los que creemos, creciente y convencido que ese es el camino. Lo demuestra el papel de nuestros investigadores y tecnólogos, respaldados por nuestros docentes, nodocentes y estudiantes, quienes cumplen un rol fundamental en esta crisis humanitaria, rodeada además de otras pestes prioritarias de resolver para nuestra región como por ejemplo el dengue, el chagas o el hambre.

3- Es una responsabilidad y objetivo fundamental interactuar con nuestros graduados emprendedores e innovadores que integren o no formalmente el sistema científico tecnológico pero que invierten esfuerzo y conocimiento en un sistema productivo nacional y regional, siempre constructor de soberanía y de una mayor equidad. Además de actualizar permanentemente sus conocimientos, procuramos igualar oportunidades y protegerlos, entusiasmarlos e incentivarlos ofreciéndoles nuestra infraestructura, nuestro equipamiento sofisticado (que se vuelve obsoleto antes de volverse viejo, muchas veces subutilizado si su único rol es la enseñanza y la investigación), nuestros vínculos, promoción y avances, sin abandonarlos ni tomar distancia una vez concluido el ciclo formativo formal. La Universidad tiene múltiples relaciones con el sistema productivo e institucional regional y nacional, pero el que debe conformar con sus graduados debe ser nítido y contundente. Nuestro país y nuestra región necesita de profesionales, de recursos humanos formados para la ciencia y el desarrollo tecnológico y también para ser capaces de insertarse en el tejido productivo y de servicios indispensables para un país más soberano y para una sociedad más integrada. La Universidad Pública y sus graduados son eslabones de una misma cadena que será robusta e indestructible si comprende que su alianza permanente en la formación, el 
trabajo y la producción, es desarrollo generalizado, fortaleza en las circunstancias amenazantes y certeza de progreso colectivo.

4- La UNLP se obliga, con vocación infinita, a integrar la red social en todos sus niveles e interactuar naturalmente con la comunidad, intercambiando saberes y miradas, como parte de un proceso formativo recíproco, especialmente con aquellos conciudadanos que tienen menos y sufren más necesidades. La extensión universitaria como parte del proceso formativo de estudiantes y docentes debe ocupar un lugar de jerarquía que se ensanche con la capacitación formal alternativa de aquellos sectores de la comunidad que precisan adquirir y certificar conocimientos y habilidades que multipliquen sus oportunidades en el mundo del trabajo y formalizar y fortalecer capacidades comunitarias para mejorar sus condiciones de vida. La universidad pública debe saber armonizar en su actividad la educación formal de pregrado, grado y posgrado, la producción científica, tecnológica y artística, la transferencia de sus frutos y su integración académica a la región y al mundo, con la necesaria cercanía a las necesidades coyunturales y estructurales del pueblo del que proviene.

La inmensa mayoría de nuestros conciudadanos con necesidades de más conocimientos para vivir mejor -sobre todo los jóvenes, pero no sólo los jóvenes- no busca en la Universidad un título de grado. No quiere necesariamente ser ingeniero o filósofo o geólogo, pero sí mira a la universidad pública como fuente de oportunidades, demandándole conocimientos para insertarse mejor en su medio.

El aula universitaria, el taller, el laboratorio tienen tiempo y espacio disponible para poblarse de ciudadanía con voluntad y entusiasmo por aprender siempre y a toda hora. Esto nos consolidará como una sociedad más fuerte, hábil, sabia, 
capaz de entender que las oportunidades están hasta en los tiempos más difíciles y debemos saber encontrarlas.

5- Además, nuestra Universidad debe responder a las demandas institucionales crecientes que le hace la sociedad y crecer en diversidad, flexibilidad y articulación. Formar parte de la comunidad académica regional y mundial, intercambiar saberes y experiencias, integrar redes universitarias formativas, científicas y solidarias que nos permita estar a la altura de nuestro tiempo y comprender los escenarios en los que nos toca evolucionar, movilizando estudiantes, profesores e investigadores en un intercambio productivo que sume siempre en la interacción; y además integrar redes de instituciones no universitarias, comunitarias, profesionales -especialmente de nuestros graduados-, siendo rigurosos en la prioridad de la selección, conscientes que pertenecemos y construimos un modelo que tiene como objetivo fundamental, el esfuerzo de lograr la inclusión social generalizada.

Sobre estas bases asentamos nuestra reacción y nuestras estrategias académicas, pero también científicas y tecnológicas y de compromiso institucional ante la circunstancia de esta pandemia.

\section{Las prioridades en la UNLP}

Una institución educativa de gran complejidad como la Universidad Nacional de La Plata afronta y desarrolla centenares de programas y proyectos emergentes de políticas académicas, científicas y sociales que trazan objetivos acordes con las definiciones expuestas. El Plan Estratégico de la UNLP, instrumento de gestión participativa emergente de un proyecto institucional que comenzó a sistematizarse como herramienta en el año 2004, cuenta hoy con más de 1.200 programas y proyectos que se renuevan con distinta periodicidad. 
Todos coadyuvan a consolidar el modelo, los objetivos y las estrategias 0 caminos expuestos para poder alcanzarlos. La mayoría estuvo siempre presente en el Plan, sin embargo, su concreción o la celeridad y profundidad de los cambios de escenarios en todas las escalas, exigen y definen prioridades. Este es un tiempo para gestionar por prioridades y las circunstancias inesperadas que le impone a la Educación Superior una pandemia mundial reafirman esta condición:

1- El mayor rendimiento académico y el incremento de la graduación en tiempos proporcionados con la vida útil de cada ciclo formativo son una condición imprescindible para situar a nuestra universidad como una institución útil a la demanda insistente de un país y una sociedad que nos sostiene. Debemos atender las razones particulares y generar políticas para resolver las razones generales. No podemos darnos el lujo como institución y como país, de tener un desgranamiento estudiantil feroz, una duración laxa de las carreras ni de un raquítico nivel de egreso. No nos lo debemos permitir.

La pandemia 2020-21 sólo vino a reafirmar esa visión. La mejora del rendimiento académico y del egreso se basan en la voluntad del estudiante de hacer mayores esfuerzos para avanzar en su carrera, pero ofreciéndole más opciones que las que tenía hasta ahora.

Implementamos una ampliación del calendario académico con los estudiantes de grado activos. Todavía en muchos casos, un tercio del año el docente se separa del estudiante, salvo para tomarle algún examen. Los cursos de ingreso y de adaptación a la vida universitaria ahora empiezan en la segunda quincena de enero y no en abril, en la mayoría de las facultades. Si las cursadas normales empiezan en abril, entonces febrero y marzo son meses de cursadas intensivas de verano y recuperatorios. Las vacaciones de julio y las de verano 
sirven para recuperar parciales, reforzar conocimientos, completar cursadas que quedaron incompletas, tener mesas de consulta para aclarar dudas previo a los exámenes, hacer cursos integradores que reemplacen al examen si la cursada está aprobada o preparatorios de repaso previos al examen. Incluso para cursar en forma intensiva materias cuatrimestrales.

Pasamos de un calendario de 8 a uno de 11 meses con el alumno con voluntad de estudiar en el aula. Las materias tienen en su inmensa mayoría, un sistema promocional alternativo al examen final para toda la cursada, más exigente en la aprobación de las cursadas, pero dando la alternativa al muchas veces traumático examen final. Los tutores alumnos avanzados y los docentes tutores hacen un acompañamiento personalizado del estudiante en la masividad durante toda la carrera, poniendo énfasis en el año de ingreso y en el trabajo final de carrera. Si las materias son cuatrimestrales, deben poder brindarse en los dos cuatrimestres. Debemos recuperar a los estudiantes avanzados que por alguna causa dejaron la carrera con más del $80 \%$ de las materias aprobadas. La sociedad invirtió mucho en ellos para que puedan ser profesionales.

La tecnología necesaria para la educación virtual forzada en el 2020-21 avanza para transformarse en un complemento definitivo de la educación y la evaluación presencial que permita más inclusión en esta universidad masiva.

Empezamos esta gestión en junio de 2018 y aunque el calendario académico se mide del $1^{\circ}$ de abril al 31 de marzo, en junio de 2021 llevamos tres años de implementación paulatina de este ensanche de oportunidades para el estudiante con voluntad de avanzar en su carrera invirtiendo un mayor esfuerzo y los resultados son muy importantes: en marzo de 2021 (ciclo 2020) los estudiantes aprobaron 75.591 materias más en el año, que en ciclo 2017, último con el sistema anterior (que cerró en marzo del 2018) a pesar de la 
pandemia. Esto es un $26,6 \%$ de crecimiento en materias aprobadas en tres años y con un sistema que recién empieza a implementarse y que crece permanentemente.

La pandemia nos encontró en medio de este proceso y nos impuso un nuevo desafío: la adecuación a la educación virtual en nuestros cinco colegios con sus 5.000 estudiantes y en nuestras 17 facultades con sus 115.000 estudiantes de grado.

Compramos patentes, utilizamos software libre, acompañamos la gestión generalizada de la gratuidad de los pulsos telefónicos, aprovechamos nuestras plataformas. Cada Facultad, Colegio y Cátedra fue encontrando la modalidad que le resultaba más cómoda y funcional. También nos capacitamos fuertemente en cada una de esas modalidades. Desde marzo de 2020, las primeras 20 semanas dimos un curso virtual de capacitación a nuestros docentes que seguimos complementando y actualizando A cada curso se anotan y asisten virtualmente miles de docentes.

En la UNLP funcionan 3.270 cátedras de grado. Entre las cuatrimestrales y las anuales, en el primer cuatrimestre deben funcionar 1.975 y funcionaron virtualmente en el 2020, 1.916 -el 97\%-. Lo mismo sucedió en el segundo cuatrimestre. Desde ya que tenemos problemas, pero estamos aprendiendo y los estamos resolviendo.

Equipamos nuestro taller de recuperación de computadoras, tablets y notebooks, que acepta miles de donaciones de artefactos en desuso de instituciones y particulares y las actualiza y repara, y además compramos tablets, lanbooks y notebooks (hasta ahora unas 2.000) para dotar de 
tecnología a aquellos estudiantes que no tienen ni un celular. Son miles y los artefactos son caros, pero lo estamos logrando.

Desde la perspectiva del Día Después, la virtualidad va a cumplir un rol complementario mucho más importante del que cumplía antes de la pandemia. La presencialidad se va a enriquecer. Por lo pronto, estamos transitando la crisis avanzando por el mismo camino y con el mismo objetivo prioritario: incluir a todo estudiante que esté dispuesto a hacer el esfuerzo y avanzar.

La consecuencia directa es el acercamiento entre la duración real de las carreras a la duración teórica y el crecimiento en la cantidad de graduados. Cambiamos el paradigma del funcionamiento en el grado. La conclusión elemental es que cuando el Estado está presente exigiendo esfuerzos individuales y colectivos, pero ofreciendo oportunidades de progreso, la Comunidad las aprovecha y la Sociedad en su conjunto se beneficia.

Por su lado, las prioridades para las nuevas carreras de grado en una Universidad prolífica en su oferta, están reservadas a las demandas acuciantes de una sociedad en transformación y a las necesidades de estar a la altura de un rumbo mundial de producción de nuevos conocimientos y tecnologías. Mucho tenemos para hacer en la adecuación a las demandas contemporáneas de nuestro actual sistema.

2- El crecimiento de los desarrollos científicos y tecnológicos enfocados en las necesidades sociales, el cuidado ambiental y el desarrollo soberano de nuestro país ocupan un lugar central en nuestras políticas. Como universidad pública tenemos que ser y demostrar ser una herramienta imprescindible para el progreso colectivo nacional y para eso debemos producir conocimiento útil y transferirlo intensamente a las instituciones de la Sociedad Civil, a la micro, pequeña y mediana empresa, al Estado en todos sus niveles, sin embargo, el 
crecimiento y la protección firme de nuestros recursos humanos formados son la única garantía para que podamos seguir dando respuesta al sinnúmero de demandas sociales e institucionales a la ciencia y a la tecnología.

Nuestros programas apuntan a garantizar el funcionamiento de nuestras unidades de investigación y transferencia (cercanas a las 200 incluyendo 150 laboratorios, Centros e Institutos), en dotarlas de infraestructura y equipamiento adecuado, de asegurarnos que esos equipamientos sean reparados cuando tienen alguna dificultad (y esos significa muchas veces grandes esfuerzos), pero fundamentalmente se enfocan en nuestros recursos humanos: que cuenten con las becas y subsidios elementales para sostenerse en el sistema y seguir produciendo ciencia, haciendo todo lo posible para brindarles estabilidad y máxima dedicación, protegiéndolos en las épocas en las que otros sectores del Estado los rechazan o expulsan, ayudándolos en sus viajes de estudio en épocas de normalidad, en los eventuales costos para publicar, en la organización, gastos y logística de encuentros de intercambio, hoy virtuales pero ayer y mañana presenciales o mixtos.

En esta pandemia el aporte de nuestros investigadores en las tareas de diagnóstico, en avances científicos funcionales y convergentes a la causa de la crisis y en el voluntariado social en los más diversos campos, es formidable.

3- Insistimos en afirmar que es imprescindible involucrarnos con el sistema productivo y de servicios, sobre todo a partir de nuestros graduados emprendedores e innovadores, articulando con ellos nuestro inmenso dispositivo de infraestructura, equipamiento y producción de nuevos conocimientos. Poniéndolo a disposición de facilitar y acelerar su creatividad, su cultura emprendedora y su capacidad de asociarse produciendo sinergias mucho más potentes que las posibles desde su esfuerzo aislado e individual, 
constituyéndonos en núcleos de vinculación complejos y útiles al progreso de quienes formamos. Esto promueve un efecto multiplicador que acentúa el perfil de una región como la nuestra, que se caracteriza por una alta proporción de población formada y que no tiene activos naturales, ni humanos que tengan ni cerca, la dimensión del conocimiento.

El paso en esta etapa es lograr la construcción de Centros Logísticos Tecnológicos y de Extensión dotados de equipamiento y fibra óptica potente que además complementen la actividad formativa y de investigación de cada Facultad en las más diversas ramas del conocimiento.

Construimos un Centro Informático de la Facultad homónima asociado a grandes y hasta micro empresas nacidas en la Universidad -más de 70- y los resultados son muy alentadores. Un Centro de Desarrollo Tecnológico FAUtec- en la Facultad de Arquitectura y Urbanismo, un Centro de Extensión (en calle 2 entre 43 y 44) de la Facultad de Psicología, una Escuela de Oficios que lidera la Facultad de Trabajo Social, un Centro Público de Arte que dirige la Facultad de Artes que también cuenta con un moderno set de cine y un taller de escenografía, un Hotel Escuela para la carrera de Turismo de la Facultad de Ciencias Económicas (42 habitaciones dobles con auditorio para 180 personas y aulas), un Hospital Integrado con alta tecnología de la Facultad de Medicina.

La Facultad de Ciencias Astronómicas y Geofísicas cuenta con el Planetario y coordina el Centro Interdisciplinario de Investigaciones Aplicadas al Agua y al Ambiente -CIIAAA- acompañada por las Facultades de Ingeniería y la de Ciencias Naturales y otras universidades e instituciones científicas. La Facultad de Ciencias Naturales cuenta con el Museo de La Plata, de Ciencias Naturales. La Facultad de Humanidades y Ciencias de la Educación, en las carreras de Educación Física, cuenta además de su propio complejo, con el Centro de 
Educación Física de la UNLP, la Facultad de Ingeniería cuenta con el complejo del "Instituto Malvinas" pero participa de múltiples actividades con otras Facultades, la Facultad de Periodismo y Comunicación Social lidera el funcionamiento del Canal, la Radio AM y FM y la Editorial de la UNLP y realiza una intensa tarea de extensión y voluntariado en su propia sede y en diversos centros periféricos al igual que la Facultad de Ciencias Jurídicas y Sociales.

Las Facultades de Ciencias Agrarias y Forestales y de Ciencias Veterinarias comparten varios campos y centros experimentales. La primera además cuenta con el Centro Tecnológico de la Madera orientado a construir todo tipo de piezas pero en particular unidades habitacionales y muebles para la emergencia social y con un centro de producción de alimentos deshidratados 150.000 raciones diarias de legumbres, hortalizas y carnes- para contribuir a dar respuesta a la emergencia social, apuntalado fuertemente por el CIDCA, Centro de Investigación especializado en alimentos de la Facultad de Ciencias Exactas, articulado con nuestro importante corredor hortícola, ayudándolos con la deshidratación de sus excedentes, los que siempre fueron desechados. Esta Fábrica Pública de Alimentos Deshidratados que se transformará en el núcleo de un sinfín de emprendimientos alimenticios con el valor agregado de nuestros conocimientos: prebióticos, probióticos, kéfir, subproductos de la horticultura regional y tantos otros que conocemos y que aparecerán.

Veterinarias comparte con otras facultades múltiples proyectos y cuenta con el Hospital de Grandes y Pequeños Animales.

Seguiremos con Energía alrededor de YTEC una empresa pública potente, de YPF y el CONICET, que logramos que se asentara en una parcela proporcionada por la UNLP que forma parte de un predio mucho mayor. También en ramas del conocimiento en donde tenemos fortalezas que ofrecer: 
agua, alimentos, medicamentos y vacunas, industrias culturales, de la madera para vivienda social y muebles, entre otras que se van conformando desde nichos más específicos.

Estamos construyendo un complejo edilicio de $4.400 \mathrm{~m} 2$ que encabeza la Facultad de Ciencias Exactas pero que complementa con las facultades de Ingeniería, Medicina, Veterinarias entre otras, destinado a alojar desarrollos y producciones de un alto valor agregado de conocimientos. Entre ellas un centro para la investigación, desarrollo, evaluación y sobre todo producción de vacunas, articulado con el Instituto Biológico Dr. Tomás Perón de la Provincia de Buenos Aires, Y una Fábrica de celdas y baterías de litio, articulados con y asociados a Y-TEC, INVAP, Conicet, Ministerio de Ciencia y Tecnología, Ministerio de Transporte y Ministerio de Defensa de la Nación.

El Hospital Odontológico Universitario de esa Facultad es el más importante del país, con 200 sillones en sala y 60 unidades de atención periféricas a las que se suman los 16 sillones en consultorios del ex Sanatorio de la Carne de Berisso, dotado de tecnología de última generación -totalmente gratuito atiende hasta 2.000 personas diarias- es un ejemplo de solidaridad, pero también de sinergia y articulación con sus graduados.

El sistema de enseñanza en Ciencias de la Salud es una particularidad. Lo integran 14 carreras de diferentes facultades con unos 20.000 estudiantes y 3.000 docentes. Avanza en un sistema integrado de prácticas denominado Hospital Público Universitario en red, con el sistema de salud pública regional tanto provincial como municipal, integrado por 7 hospitales públicos de alta complejidad, 14 de complejidad intermedia y 60 unidades de atención primaria a la salud. La práctica se inicia desde el primer año del grado. 
Todos estos dispositivos se sumaron a las políticas públicas diseñadas para combatir esta pandemia. Nuestros investigadores, en centros de las Facultades de Ciencias Exactas, de Ciencias Médicas y de Ciencias Veterinarias hacen un aporte fundamental en los diagnósticos de Covid y ya están investigando una vacuna que combata ese virus. Nuestros tecnólogos fabrican máscaras, barbijos complejos, respiradores, piezas para reponer las que se rompen y hasta camas para los hospitales de campaña. Y también miles de litros de alcohol en gel, alcohol al 70\%, lavandina y jabón líquido para repartir en los barrios. $Y$ nuestros extensionistas realizan tareas de voluntariado en toda la región, acompañando a nuestra comunidad en contención y evaluación casa por casa y desde los vacunatorios que se abrieron en edificios de la UNLP. La lucha contra la peste da testimonio de la importancia de que la universidad pública sea una pieza del sistema productivo de las más diversas maneras.

4- La capacitación extracurricular formalizada que acerque y nos acerque e integre a sectores de nuestra comunidad que siempre estuvieron alejados de la universidad pública, sin advertir (ambos) que podemos ser el soporte sobre el cual puedan asentar sus expectativas de progreso o la satisfacción de sus inquietudes sobre determinados conocimientos, es el desafío más importante de nuestro tiempo para la extensión universitaria. Debemos adecuar los criterios de la educación formal en todos sus niveles a las demandas de nuestra sociedad en este tiempo, pero existe un sector enorme de esa sociedad que no recorrerá ese camino y sin embargo necesita de nosotros y de lo que sabemos hacer y requiere que lo compartamos y comprendamos lo que ellos saben y pueden hacer. Esa conjunción de saberes nos dará la oportunidad de consolidarnos como universidad popular, naturalizada en la vida cotidiana de nuestro pueblo. La construcción de esa política no solo nos llevó a 
construir una Escuela - Fábrica de Oficios, sino a ofrecer saberes y capacitaciones alternativas a un colectivo social que crece de a miles por año. El último relevamiento anual sumó unas 40.000 personas formándose en estas habilidades en nuestra universidad pública !!!

Una muestra puntual fue la convocatoria 2021 a sólo 12 talleres virtuales de oficios en los que se inscribieron 43.000 vecinos en sólo 3 días.

La enseñanza de oficios y el dictado de cursos que amplíen los conocimientos de nuestra sociedad de acuerdo a sus necesidades e inquietudes, acentúa la naturalización de la institución universitaria en sectores que no registran o no encontraban un sentido firme de vincular sus vidas con la universidad pública.

5- La construcción e integración de redes sociales con los distintos actores institucionales formales y no formales de nuestra región, nuestra consolidación como ámbito de debate e instrumento de acción colectiva y la multiplicación de vínculos activos y productivos con los distintos espacios de educación superior y ciencia en la región y el mundo, consolidando una agenda de intercambio orientada a la convergencia de necesidades concretas para alcanzar los objetivos trazados conforman un núcleo de actividades que debe consolidarse y crecer, y que se ponen en valor en estas circunstancias críticas. La cohesión social es el amalgama de sus instituciones y de sus organizaciones en general. La UNLP consolidó importantes redes de vinculación en su región como el Consejo Social, el Consejo Consultivo de Instituciones de la Comunidad, el Consejo Consultivo de Colegios Profesionales y el Consejo Consultivo Empresario, además de vínculos estrechos con Organizaciones de Derechos Humanos, de la Tercera Edad y de Organizaciones de Base. Trabaja articulada con los Gobiernos Locales, Provinciales y Nacional. En su macroregión sus vínculos y participación crecen en redes de universidades como el Grupo 
Montevideo, UDUAL y Macrouniversidades. En el mundo interactúa en el campo académico y científico con numerosas universidades en proyectos de intercambio de estudiantes, docentes y científicos e investigaciones comunes, manteniendo su visión integradora en la convergencia de saberes que consoliden su compromiso con el fortalecimiento de la agenda social, ambiental y de desarrollo soberano.

Hoy la agenda es virtual pero sólida. Y seguramente el futuro propondrá una convergencia de las tecnologías y la presencialidad para reconfigurar la agenda universitaria mundial. Por lo pronto, nosotros vamos en esa dirección.

También el crecimiento de los servicios a los estudiantes que ayuden a su integración armónica a la vida universitaria y su sostenimiento, la continuidad del plan de obras y equipamientos, el mejoramiento constante de los sistemas de administración y finanzas, los beneficios, estabilidad y acompañamiento a la comunidad trabajadora docente y nodocente, la atención especial a la mujer, los derechos humanos y en su contexto la discapacidad y la importancia creciente social, académica y científica de comprender holísticamente a la salud, así como cada una de las actividades que nos transforma en una institución activa y en crecimiento sostenido, son fundamentales en nuestra agenda que se acentúan en la actual circunstancia.

Sin embargo, todas estas son condiciones que debemos reunir para asumir con más fuerza la responsabilidad máxima de ser una institución comprometida en su presente y en su futuro con la construcción de oportunidades para nuestra gente y de alternativas de progreso para nuestra patria.

El modelo centenario de la Reforma Universitaria tal como la vivimos hoy, promueve un conjunto de principios y condiciones que nos definen como 
Institución, pero es un proceso en constante evolución acompañando las demandas de una sociedad que también evoluciona vertiginosamente, más allá de la pandemia. Cada momento de nuestra historia se configura en un escenario distintivo y cada escenario ordena las prioridades de ese proceso según las circunstancias que ordenan nuestras más profundas convicciones con el tiempo que nos toca vivir. Comprender estas condiciones es nuestra oportunidad de ser realmente útiles al progreso de nuestra sociedad.

Nuestro Proyecto Institucional reafirma nuestros principios y consolida con fundamentos sólidos su defensa, dándole al debate general el respaldo necesario para poder afrontar un presente desafiante y crítico e imaginar responsablemente un futuro deseado, con la certeza que estamos respaldados por la mirada atenta y lúcida de nuestra sociedad y nuestros pares, que nos alerta sobre cada intento de desvirtuar nuestra esencia y fundamenta las aristas más sutiles que nos permiten avanzar en la construcción de una universidad comprometida con su historia y con una Comunidad que la reclama. Ese debe ser nuestro compromiso, cortar la maleza que nos enreda, lastima y demora y avanzar, siempre avanzar.

\section{Algunas cifras orientativas de la evolución de la UNLP desde el inicio de su Plan Estratégico}

En los 9 rankings mundiales que aparecieron en el 2020 /2021, la UNLP fue ubicada 2a ${ }^{\text {- }}$ en la Argentina, entre las 10/20 (en uno 26) más importantes en América Latina y el Caribe de las hasta 4.000 universidades registradas en el subcontinente y entre las 500/600 más importantes en el mundo entre las hasta 30.000 universidades registradas. 
En el ránking de impacto de la producción científica julio 2020 medida en citas a la producción de sus investigadores, la UNLP "con 1.129.966", se ubica $1^{\text {a }}$ en Argentina, $3^{\text {a }}$ en América Latina y el Caribe y 179 en el mundo, sobre un total de 3.745 instituciones de educación superior (de un universo de 30.000) que cumplieron con la condición mínima y básica de superar las 1.000 citas en toda la universidad.

La UNLP contaba con 40.000 integrantes -estudiantes y trabajadores docentes y nodocentes- en el período normalizador democrático de 1984. En marzo del 2021 ronda los 200.000 discriminados en 5.000 estudiantes de educación inicial, primaria y secundaria; 110.000 estudiantes de grado, 15.000 estudiantes de carreras de posgrado, 10.000 estudiantes de cursos de posgrado, 5.000 estudiantes de oficios, 35.000 estudiantes en cursos alternativos a la formación de grado, 3.600 no docentes y 14.000 docentes.

La UNLP pasó en los últimos 10 años de 4.000 a 6.500 graduados en el grado. Proyecta, con las medidas en marcha, llegar a los 10.000 graduados anuales en los próximos 5 años sin crecer en cargos docentes sino reforzando el salario por una mayor carga horaria de quienes se sumen al Programa de Promoción del Rendimiento Académico y el Egreso -PRAE-. La relación egreso 2017 referida al ingreso 2011 fue del 44\% (era del 25\% hace una década). Aspiramos a llegar al $60 \%$ en el período expuesto.

La educación formal alternativa a la formación de grado era inorgánica e incipiente hace diez años. Hoy suma 5.000 estudiantes de oficios en cursos de hasta dos años y otros 35.000 en otros refuerzos de conocimientos no convencionales. 
Como referencia, en marzo de 2021 -en medio de la pandemia del Covid- se llamó a inscripción a 12 cursos a distancia con una mínima carga presencial y se anotaron 43.000 personas !!!

Las redes institucionales microregionales de la UNLP integran 500 instituciones de la Sociedad Civil y organizaciones de base nucleadas en Consejos Consultivos y el Consejo Social. El trabajo en red es cotidiano.

El sistema científico tecnológico de la UNLP comprende 151 Laboratorios, Centros e Institutos de Investigación y 50 Unidades independientes que concentran unos 7.000 investigadores y becarios que acreditan unos 750 proyectos de investigación por año y producen más de 5.000 publicaciones indizadas.

Desde que pusimos en marcha el Plan Estratégico de la UNLP en el año 2004 construimos unos $180.000 \mathrm{~m} 2$ y recuperamos, restauramos y pusimos en condiciones $150.000 \mathrm{~m} 2$ de los 310.000 que existían hasta ese momento (55\% de $\mathrm{m} 2$ nuevos y casi $50 \%$ de $\mathrm{m} 2$ reacondicionados). Se construyeron y recuperaron aulas, laboratorios, bibliotecas, facultades enteras, colegios y numerosas infraestructuras especiales: un planetario, un centro de convenciones y eventos para 4.000 personas, un centro de arte, un hospital odontológico escuela con 200 sillones (más 70 sillones externos), una unidad de producción social de medicamentos y vacunas, un laboratorio de análisis clínicos gratuitos y una unidad de producción de anteojos gratuitos; un hotel escuela universitario para 84 personas (42 habitaciones, auditorio y aulas), un centro de posgrado, centros de innovación tecnológica, una escuela de oficios, un albergue, comedores universitarios, un set de cine, uno de televisión, un canal de aire, dos radios, una fábrica para el tratamiento de la madera equipada para la construcción de viviendas sociales y muebles y hasta un 
Banco Público. También un Hotel Escuela, una fábrica social de alimentos deshidratados (con deshidratadoras), articulada con frigoríficos y con el cinturón hortícola de la región) con una capacidad de 150.000 raciones diarias. Estamos en plena construcción de un centro de investigación, desarrollo, control y producción de vacunas y de una fábrica de celdas y baterías de litio. Además, entre sus infraestructuras especiales, la Universidad cuenta con 17 museos y 23 bibliotecas.

Los servicios estudiantiles sumaron la reapertura después de 30 años del Comedor Universitario con turnos al mediodía y viandas nocturnas al que asisten un promedio de 10.000 estudiantes diarios. Es gratuito para el estudiante de escasos recursos y con un costo de $\$ 50$ en 2020 para el resto.

También sumamos el Albergue Universitario para 200 estudiantes sin recursos, que no podrían acceder a los estudios universitarios sin la asistencia total de nuestra universidad pública. Las habitaciones son individuales y ya superamos las 150 ocupadas. El requisito de ingreso son sus dificultades económicas extremas y de permanencia es acreditar la aprobación del 17\% de las materias de su carrera por año. Se recibe el $90 \%$ de los ingresantes en las más diversas disciplinas.

Pusimos en marcha una línea de micros universitarios denominada "Rondín Universitario" que une el centro de la ciudad con la puerta de las 17 facultades y 3 colegios urbanos de la UNLP. También un Tren Universitario, recuperando $5 \mathrm{~km}$ de vías existentes y construyendo 6 paradores que contribuyen con el transporte público de universitarios y de toda la sociedad. Acabamos de firmar un convenio con el Ministerio de Transporte para ampliar $8 \mathrm{~km}$ más con 8 paradores el recorrido original. También contamos con dos rondines de "Ecobus" (una especie de micros urbanos gratuitos que funcionan con baterías 
de Litio) y entregamos anualmente centenares de bicicletas estudiantiles en comodato hasta el final de la carrera.

Participamos en la redacción y fuimos la única universidad que gestionó para todos la Ley del Boleto Estudiantil en la Provincia de Buenos Aires (14.735). Con una fuerte participación estudiantil, en múltiples marchas y acciones, conseguimos la aprobación unánime en ambas cámaras de la Legislatura Bonaerense. El 16 de septiembre de 2016, día del 40 aniversario de "La Noche de los Lápices" (hecho luctuoso en épocas de la dictadura militar donde una decena de estudiantes secundarios - la mayoría de la UNLP- fue torturado y muerto por reclamar el boleto estudiantil) el transporte público urbano empezó a recoger estudiantes universitarios con 45 pasajes mensuales gratuitos y la totalidad de los estudiantes de educación primaria y secundaria de toda la provincia y de otras universidades en forma gratuita.

También fuimos la única Universidad que gestionó en forma constante hasta conseguir su sanción y promulgación la Ley Nacional 27.204 de reforma de la Vieja Ley de Educación Superior 24.521/95 a partir de la cual reafirmó la autonomía universitaria y se garantizó el ingreso irrestricto y la gratuidad de los estudios universitarios en todo el territorio nacional. La Ley fue sancionada el 28 de octubre y promulgada el 9 de noviembre de 2015.

\section{Bibliografía}

(Principales antecedentes, enfoques conceptuales y avances del Plan Estratégico de la Universidad Nacional de La Plata)

- Tauber, F. (2009). Comunicación en la planificación y gestión de las universidades públicas argentinas: El caso de la UNLP en el trienio junio 2004-mayo 2007. 
- Tauber, F. (2010). Discurso de asunción a la Presidencia de la UNLP.

- Tauber, F., Nizan, G., Delucchi, D., \& Olivieri, A. (2011). Planes directores, proyectos y obras. 2004-2010. Universidad Nacional de La Plata (UNLP).

- Tauber, F. (2011). Universidad pública y medioambiente: responsabilidades y desafíos para los próximos años. Revista de la Universidad.

- Tauber, F. (2011). Pensar la Universidad Nacional de La Plata 1 (20102014). Universidad Nacional de La Plata (UNLP).

- Tauber, F., \& Urriolabeitia, V. (2012). UNLP:" Pensada y creada para promover el desarrollo nacional". Desafío Empresario, 10.

- Tauber, F. (2012). Inmensas oportunidades. Diario Hoy en la Noticia.

- Tauber, F. (2013). 30 años de democracia en la universidad pública. De Los Cuatros Vientos

- Tauber, F. (2013). Profesionales íntegros y comprometidos. Revista de la Agremiación Odontológica de Berisso, La Plata y Ensenada

- Tauber, F. (2013). Una idea sobre los edificios y espacios de la UNLP. Construir la Universidad 1

- Tauber, F. (2013). Internacionalización de la Universidad hacia la Región Latinoamericana. El caso de la Universidad Nacional de La Plata

- Tauber, F., \& Giordano, C. J. (2014). Plan Estratégico de la Universidad Nacional de La Plata 2014-2018

- Tauber, F., Nizan, G., Delucchi, D. (2014). Planificación y gestión urbano/ambiental de los ámbitos universitarios. XI Simposio de la Asociación Internacional de Planificación Urbana y Ambiente (UPE 11) ISBN: 978-950-34-1133-9 (La Plata, 2014) 
- Tauber, F. (2015). La universidad autónoma argentina desde el contexto democrático latinoamericano.

- Tauber, F., Giordano CJ., Marengo R., Cornejo L., Virgolini MB (2015). Planeamiento de la Educación Superior: los problemas que adoptamos y supimos construir. Revista de la Facultad de Odontología 2015

- Tauber, F. (2016). Proyecto Institucional de la Universidad Nacional de La Plata (2018-2022). Pensar la Universidad

- Tauber, F., et al. "Plan de gestión de infraestructura universitaria Polo de Investigación y Transferencia Etapa I." Facultad de Ciencias Exactas UNLP (2017).

- Tauber, F. (2017). Haciendo realidad grandes proyectos. Econo 8

- Tauber, F. "Presentación del Dr." Arq. Fernando Tauber del nuevo complejo edilicio en el predio del Ex BIM III (2017).

- Tauber, F. (2017). Industrias de valor estratégico regionales, políticas institucionales de la UNLP para promover su desarrollo.

- Tauber, F. (2017). Hacia el segundo manifiesto: Los estudiantes universitarios y el reformismo hoy.

- Tauber, F. (2017). Reseña del Plan Estratégico de Obras de la UNLP.

- Tauber, F. (2017). La Reforma y el futuro de la Universidad. Revista Derechos en Acción

- Tauber, F. (2017). La Universidad Nacional de La Plata en el Centenario de la Reforma Universitaria

- Tauber, F. (2017). La Universidad Nacional de La Plata en el contexto actual: la búsqueda de un modelo inclusivo. + E 7 (7. Ene-dic), 132-143

- Tauber, F. "Discurso: Pensar la universidad." Proyecto institucional 2022 (2018). 
- Tauber, F. (2018). La universidad pública reformista: un modelo inclusivo y de desarrollo. Eudeba

- Tauber, F. (2018). Entrevista al Dr. Fernando Tauber sobre el proyecto de planta piloto E-Basura.

- Tauber, F. ( entrevista, de, Santiago, Barcos) (2018). Los dolores que quedan son las libertades que nos faltan. Econo 8

- Tauber, F., Cereijo, R., Palladino, A. (2019). Se cumplen setenta años de la sanción de la gratuidad universitaria en Argentina. Diario Contexto 22

- Tauber, F. (2019). UNLP Un Modelo de Universidad Pública Argentina. Revista Universidades

- Tauber, F., Cruz, V. (2019). El proyecto político-académico de la Universidad Nacional de La Plata y los desafíos de fortalecer la política institucional de géneros y feminismos. Gaceta de la $\vee$ Circunscripción plurinominal del Tribunal electoral del Poder Judicial de la FederaciónNueva época

- Tauber, F. (2019). La función de la universidad pública en el desarrollo comunitario.

- Tauber, F. (2019). Extensión universitaria y producción social.

- Tauber, F. Extensión universitaria: rupturas y continuidades. Educación.

- Tauber, F. (2020). Comunicado del Dr. Arq. Fernando Tauber en el marco de las jornadas de capacitación virtual para Directores de Enseñanza y Secretarios Académicos

- Tauber, F. (2020). Derecho a la educación en tiempos de pandemia. Material audiovisual aportado por el Centro de Producción Multimedial 
(CEPROM). Para acceder al video y para su mejor reproducción, hacer clic en" Enlace externo".

- Tauber, F. (2020). Modalidad virtual en la Escuela Universitaria de Oficios. Material audiovisual aportado por el Centro de Producción Multimedial (CEPROM). Para acceder al video y para su mejor reproducción, hacer clic en" Enlace externo".

- Tauber, F. (2020). Universidad Nacional de La Plata 2020 (Doctoral dissertation, Universidad Nacional de La Plata).

- Tauber, F. (2020). Palabras inaugurales del Dr. Arq. Fernando Tauber en el marco de las $3^{\underline{a}}$ Jornadas sobre las Prácticas Docentes en la Universidad Pública

- Tauber, F. (2020). Construyendo los cimientos para una segunda reforma universitaria.

- Tauber, F. (2020). Discurso del Dr. Arq. Fernando Tauber sobre "Las Acciones y el Rol de las Universidades en el Contexto de la Pandemia"

- Tauber, F. (2020). Las respuestas de la universidad pública a los desafíos actuales.

- Tauber, F. (2020). Convicción y compromiso. Maíz

- Tauber, F. (2020). La Universidad consolidando los procesos socioorganizativos de la comunidad. Radio 10

- Tauber, F. (2020). Respuesta institucional ante la pandemia. Radio Universidad

- Tauber, F. (2020). Investigación, Transferencia e Innovación productiva en políticas soberanas: los ejes de gestión de la Universidad Nacional de La Plata. 
- Tauber, F. (2020). La virtualización de la enseñanza como política de inclusión.

- Tauber, F. (2020). La capacidad productiva de la UNLP a 115 años de su fundación.

- Tauber, F. (2020). Universidad y Estado nacional.

- Tauber, F. (2020). Confluencia de la universidad y la política.

- Tauber, F. (2020). Las respuestas concretas de la Universidad a los problemas de hábitat e inequidad social en los tiempos actuales.

- Tauber, F. (2020). El desafío de gestionar en un escenario de pandemia.

- Tauber, F. (2020). Las condiciones actuales para el reformismo en la universidad pública.

- Tauber, F. (2020). Articulación del sistema científico-tecnológico y el académico para el desarrollo social de la región.

- Tauber, F. (2020). El modelo de educación pública de la UNLP.

- Tauber, F. (2020). Consejo Social de la UNLP: Una herramienta para el diseño y la aplicación de políticas de desarrollo.

- Tauber, F., \& Saintout, F. (2020). La UNLP frente al COVID-19: acciones, articulaciones y estrategias desplegadas para la prevención, monitoreo y control de la pandemia, y el fortalecimiento del sistema público de salud.

- Tauber, F. (2021). Medidas institucionales ante la emergencia.

- Tauber, F., \& Díaz, F. J. (2020). La visión de la UNIVERSIDAD NACIONAL DE LA PLATA en la promoción del desarrollo.

- Tauber, F. (2021). Fábrica Universitaria de Alimentos Deshidratados para fines sociales. Una apuesta de la UNLP en la lucha contra el hambre. 
- Tauber, F. (2021). Memoria, verdad y justicia.

- Tauber, F., \& Díaz, F. J. (2020). La visión de la UNIVERSIDAD NACIONAL DE LA PLATA en la promoción del desarrollo.

- Tauber, F. (2021). Fábrica Universitaria de Alimentos Deshidratados para fines sociales. Una apuesta de la UNLP en la lucha contra el hambre.

- Tauber, F., \& Palma, S. (2021). Avances y desafíos de un modelo de Universidad pública en tiempos de pandemia. Crónica.

- Tauber, F. (2021). Relaciones entre el sistema de Ciencia y Técnica de Argentina y las Universidades Públicas para el desarrollo industrial del litio con perspectiva soberana.

- Tauber, F. (2021). Pensar el desarrollo en el partido de La Plata, nuestra región 